\title{
Interaction of various types of bisphenols with enzymes involved in melanin synthesis
}

\author{
Harinishree Venkatesan ${ }^{1} \cdot$ Ranjani Soundhararajan $^{1} \cdot$ Hemalatha Srinivasan $^{1}$ \\ Accepted: 17 September 2021 / Published online: 6 October 2021 \\ (c) The Author(s), under exclusive licence to Korean Society of Environmental Risk Assessment and Health Science 2021
}

\begin{abstract}
Objectives Bisphenol is one of the commercial chemical compounds which finds application in the manufacture of phenol, polyesters, polycarbonates, epoxy resins, etc. Bisphenol A is the most commonly found bisphenol derivative, although there are various analogues such as Bisphenol S, Bisphenol B, Bisphenol C, Bisphenol E, Bisphenol AF, which vary with their structures. Bisphenols majorly get into us by means of food as they are found in food packaging and beverage containers and can be easily absorbed by the body. They can make way into the body systems either by direct means like skin contact or by indirect means through food that is stored in a bisphenol-coated containers. Exposure of the human system to this compound may bring about mild to adverse ailments ranging from skin irritations to infertility. Pigmentation disorder is one such health condition that occurs due to melanocyte destruction and is caused by bisphenol exposures. They interact with various proteins and enzymes that have been involved in the melanocyte synthesis pathway, thereby disrupting their normal functions. Methods In silico analysis has been done to predict the interaction and other descriptive binding parameters when the bisphenol derivatives BPA. BPB, BPC, BPE, BPS and BPAF have been docked with some of the essential proteins for melanin synthesis such as tyrosinase, tyrosinase-related protein 1(TRP-1), dopachrome tautomerase/tyrosinase-related protein-2(TRP-2). Results The interaction has been quantified with the binding energies, and the interacting amino acid residues have also been identified. The results showed strong interactions for both the proteins TRP-1 and TRP-2 with BPA. BPB, BPC, BPE, BPS and BPAF.

Conclusions The risk and threats caused by the commercial products made of bisphenol are at higher levels, and thereby, the need for a safe and desirable alternative has been insisted.
\end{abstract}

Keywords Bisphenol $\cdot$ Tyrosinase $\cdot$ Tyrosinase-related protein $-1 \cdot$ Dopachrome tautomerase $\cdot$ Melanocyte destruction

\section{Introduction}

Plastics and synthetic polymers have become inseparable with the mankind, as their usage by the people, in manifold forms such as thermoplastics, adhesives, thermosets, elastomers, fibres, have increased several times since its production in 1900s. It has been currently assumed that the production of plastics increased to nearly 60 million metric

Harinishree Venkatesan and Ranjani Soundhararajan have contributed equally to this work.

Hemalatha Srinivasan

hemalatha.sls@bsauniv.ac.in

1 School of Life Sciences, B.S. Abdur Rahman Crescent Institute of Science and Technology, Vandalur, Chennai, Tamilnadu 600 048, India tonnes in the past 5 years. These commercially undeniable polymers use epoxy, polyethylene, polyamide, polystyrene, polyester, polysulfone, silicone, phenolic and acrylic resins, etc. as the raw materials for their manufacturing. Bisphenol is one among the precursors that is found in common with polymers such as epoxy, polyester and polycarbonate resins [1] that are used for making cosmetics, food packaging materials, pet apparels, electronic goods, health care and sanitary products, sealants for dental filling, thermal and carbonless papers, pesticides, colourants in dye and ink for tattoos. General populations may get exposed to bisphenols by direct contact or when they intake food that are stored in containers coated with bisphenol compounds or when they breathe in the particles of it or when they drink water that comes from the plastic pipes, etc. People working in industries that manufacture or make use of these polymers and resins are susceptible to occupational exposure of bisphenol. An 
instant or constant exposure to these bisphenol compounds reported different health ailments ranging from slight to severe. They are known to be endocrine disruptors thereby affecting the normal functioning of the hormones and causing related disorders including hormone-mediated tumours. Also, they cause irritations in skin, eyes and have effects in blood cells. BPA has mild to adverse health effects on human starting from skin rashes to serious endocrine disorders such as infertility and hormone-related tumours [2]. BPB exhibits similar kind of endocrine-disrupting activities like that of BPA [3]. Studies also revealed that BPB indulges in cell proliferation activities and cell and can act as a possible cancer-causing agent. It has been conveyed from the study that halogen-containing BPC has an interrupting activity on DNA transcription by forming an inhibitory confirmation with the ER- $\beta$ receptor [4]. I. P. Pozdnyako et al. 2010 [5] reported that BPE showed estrogenic interference and has toxic characteristics. Hazards caused by BPS include gestational diabetes, obesogenic effects and fertility-related ailments [6]. They also stimulate hormonal breast cancers and are serious eye irritants. Study from Maćczak et al. (2017) [7] shows that BPAF has effects on the blood cells by interfering in the oxidative, apoptotic, necrotic and genotoxic activities and induced eryptosis. There is evidence that projects, bisphenol AF as an endocrine-disrupting chemicals [8]. Additional health conditions that may possibly develop due to bisphenol are pigmentation-related disorders which are characterized by the disability of melanocyte to produce melanin. Diseases of that kind include vitiligo, melanoma, etc. The melanocytes have a lifecycle that is initiated by the rise, proliferation and migration of neuronal crest cells, followed by their differentiation into appropriate melanogenic descent or linage. The specified melanoblasts proliferate and migrate under the governance of various signalling molecules such as Wnt, BMP, Kit, endothelin, and they acquire their morphology and gene expression profile of melanocytes [9]. The differentiated melanocytes start to proliferate and mature, accompanied by the melanosome formation and maturation [10]. The melanosome globules look connected to the filopodia of melanocyte dendrites. Various areas of these dendrites release the melanosome globules which will be absorbed by the microvilli of the surrounding keratinocytes [11] and undergo consequent cell death. Amino acid tyrosine will be converted into melanin via a series of chemical reactions [12]. However, melanin is also produced by other cells such as some neuronal cells, adipocytes, pigmented retinal epithelial cells which vary in their embryonic origin. There exist three different types of melanin in humans, namely eumelanin, pheomelanin and neuromelanin. Among the three types of human melanin, eumelanin and pheomelanin are the most common types. They have tyrosine as their biogenetic precursor and contrast with their chemical composition, structure and physical properties. Basically, eumelanin and pheomelanin vary in accordance to the variations between eumelanasome and pheomelanasome [13]. The ellipsoidal eumelanosomes and the spherical or irregular pheomelanasome differ in their biogenesis process that depends on the availability of the amino acids L-tyrsoine and glutathione or L-cysteine [14] (Fig. 1). Different classes of proteins each responsible for different functions play crucial role in the melanogenesis process. They include proteins that are responsible for constructing the structure called melanosome, enzymes that are needed for the formation of melanin and those that are involved in their transportation to appropriate target cells. Tyrosine, tyrosinase-related protein 1 and tyrosinase-related protein 2 are the very important enzymatic proteins which are required for the melanin to be produced. Previous in vivo study indicated a loss melanin in zebra fish embryos caused by BPA and BPF that may be caused due to the interruptions on biosynthetic enzymes tyrosinase and TRP1 [15]. In this study, an in silico analysis has been carried out by docking six different bisphenol derivatives such as BPA, BPB, BPC, $\mathrm{BPE}, \mathrm{BPS}$ and BPAF with two melanin synthesizing proteins, tyrosine-related protein 1 and tyrosine-related protein 2 with an objective to reveal and quantify the interactions between these proteins and bisphenols and thereby to bring into limelight about the lethal effects of commercial products involving bisphenol and its derivatives (Fig. 2).

\section{Results}

After successful running of AutoDock, the corresponding glg and dlg files were generated which were used for interpretation of the results. By using the dlg file, the binding conformation of the protein-ligand complex was obtained from the run with highest binding energy. The binding energy in $\mathrm{kcal} / \mathrm{mol}$ and the inhibitory constant (Ki) values have been considered in order to quantify the interaction between the protein and the ligand. The inhibitory constant is a value that shows how effectively a ligand can inhibit the

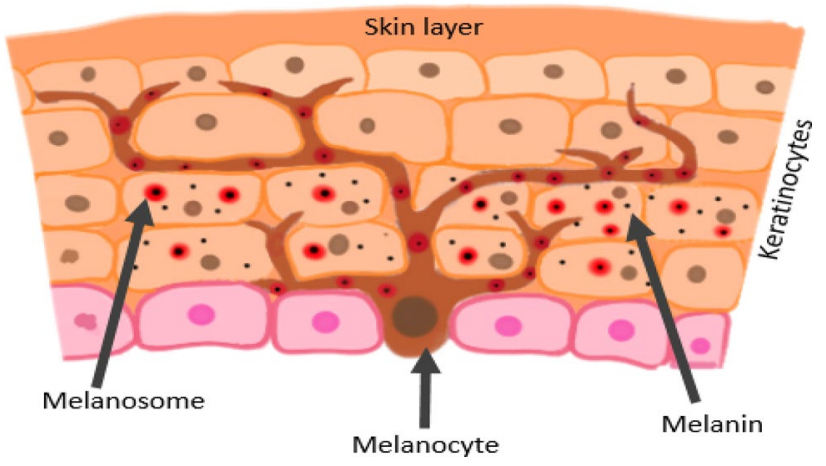

Fig. 1 Distribution of melanocytes in skin 


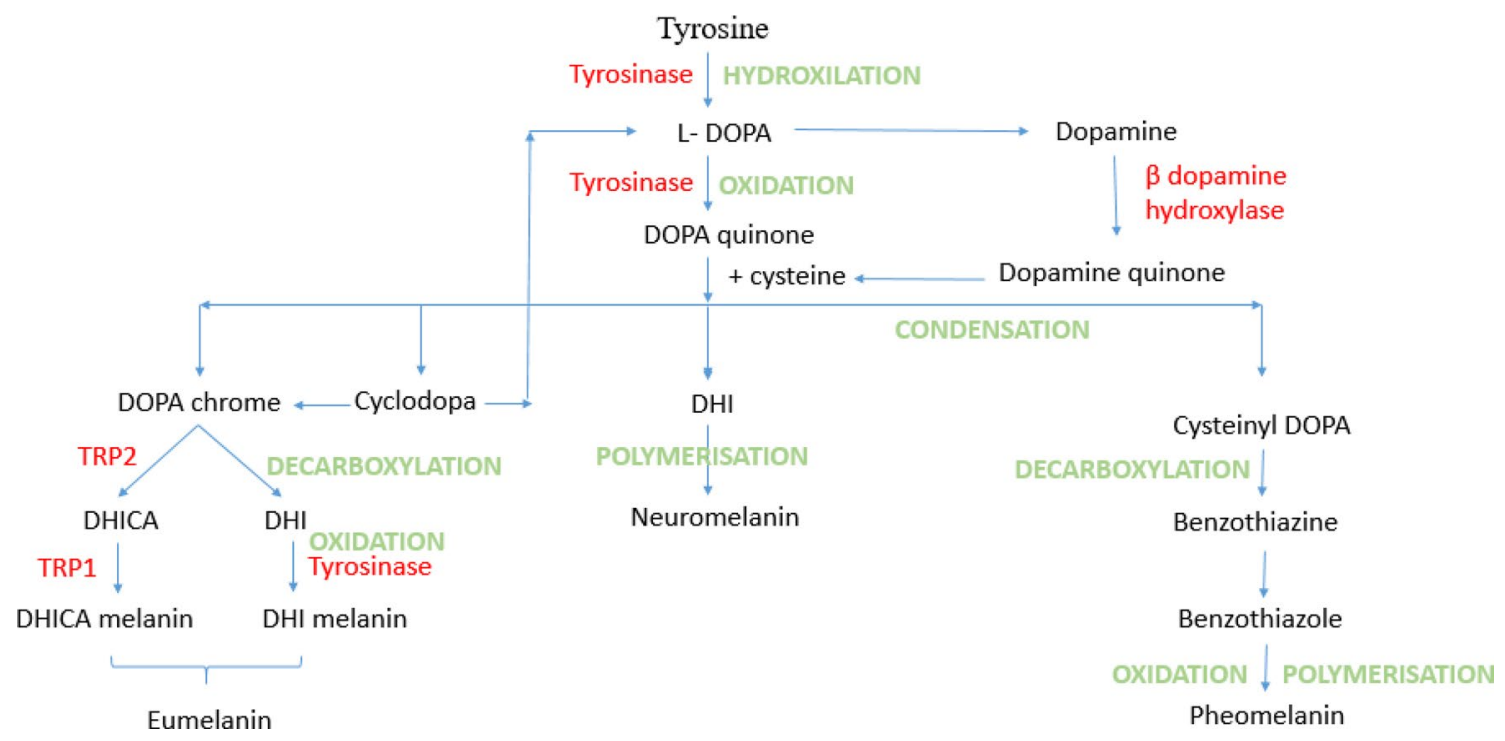

Fig. 2 Melanogenesis pathway

macromolecule. Binding energy is the energy used by the ligand to bind the macromolecule and thereby to lower the energy of the entire complex. The docked protein-ligand complex was viewed through AutoDock, where the interaction diagram was obtained for TRP 1 with BPA, BPB, BPC, BPE, BPS and BPAF are depicted in Fig. 3, and the binding energy, inhibitory constant and the interacting amino acids of TRP 1 with BPA, BPB, BPC, BPE, BPS and BPAF are depicted in Table 1. The docked protein-ligand complex was viewed through AutoDock, where the interaction pose was obtained for TRP 2 with BPA, BPB, BPC, BPE, BPS and BPAF are depicted in Fig. 4, and the binding energy, inhibitory constant and the interacting amino acids of TRP 2 with BPA, BPB, BPC, BPE, BPS and BPAF are depicted in Table 2.

Different types of bonding have been observed which included covalent bond, conventional hydrogen bonds and even some weak interactions. Highly stable covalent bonds a

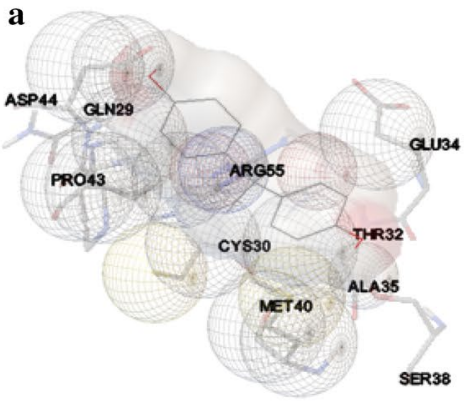

d

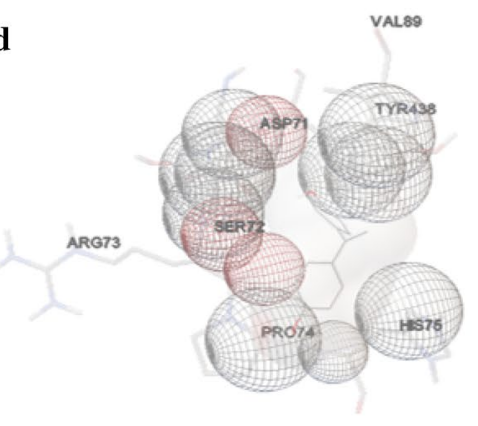

b

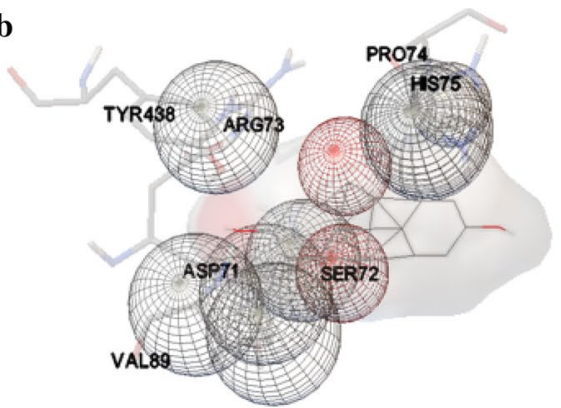

e

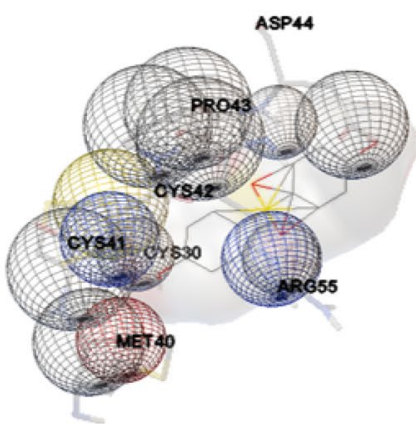

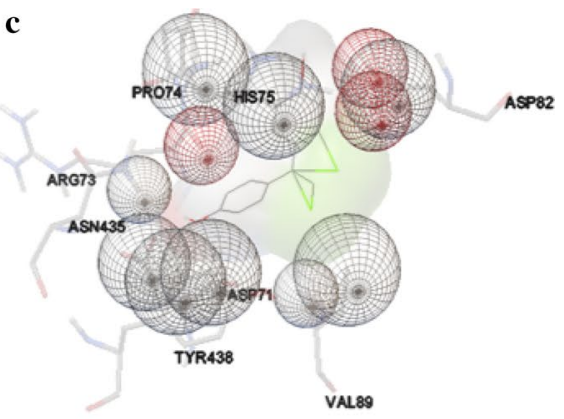

c
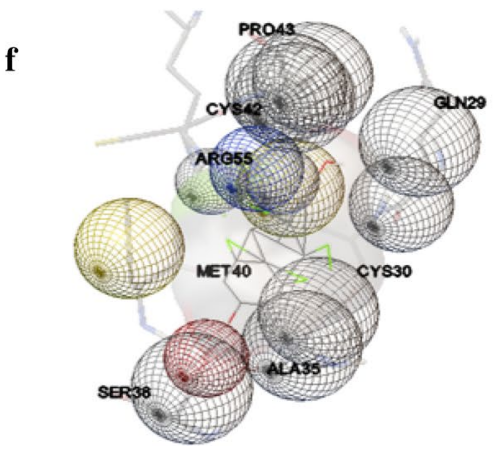

Fig. 3 Binding interactions of the protein TRP 1 with ligands: a BPA, b BPB, $\mathbf{c}$ BPC, $\mathbf{d}$ BPE, e BPS, $\mathbf{f}$ BPAF 
Table 1 Binding energy, inhibitory constant and the interacting amino acids of TRP 1 with BPA, BPB, BPC, BPE, BPS and BPAF

\begin{tabular}{llcl}
\hline Ligand & $\begin{array}{l}\text { Binding energy } \\
(\mathrm{kcal} / \mathrm{mol})\end{array}$ & $\begin{array}{l}\text { Inhibitory constant } \\
(\mu \mathrm{M})\end{array}$ & \begin{tabular}{l} 
Interacting amino acids \\
\hline BPA
\end{tabular} \\
& -5.71 & 64.98 & $\begin{array}{c}\text { ASP 44, GLN 29, PRO 43, ARG 55, CYS 30, MET 40, GLU 34, THR 32, } \\
\text { ALA 35, SER 38 }\end{array}$ \\
BPB & -7.32 & 4.32 & TYR 438, ARG 73, ASP 71, SER 72, PRO 74, HIS 75, VAL 89 \\
BPC & -7.59 & 2.73 & PRO 74, HIS 75, ASP 82, ARG 73, ASN 435, TYR 438, VAL 89, ASP 71 \\
BPE & -6.68 & 12.77 & VAL 89, TYR 438, HIS 75, PRO 74, SER 72, ASP 71, ARG 73 \\
BPS & -7.25 & 4.82 & ASP 44, PRO 43, CYS 42, CYS 41, CYS 30, MET 40, ARG 55 \\
BPAF & -6.01 & 39.14 & PRO 43, CYS 43, ARG 55, MET 40, CYS 30, GLN 29, ALA 35, SER 38 \\
\hline
\end{tabular}

a

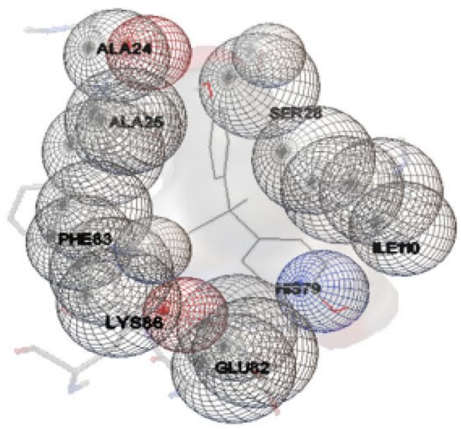

d

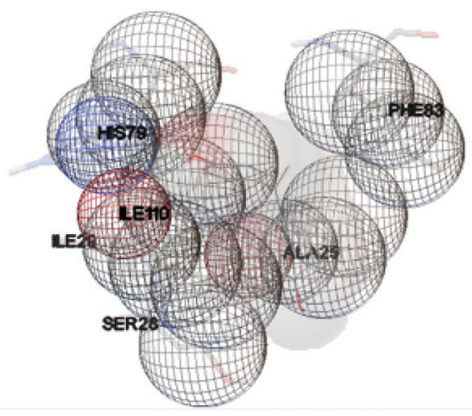

b
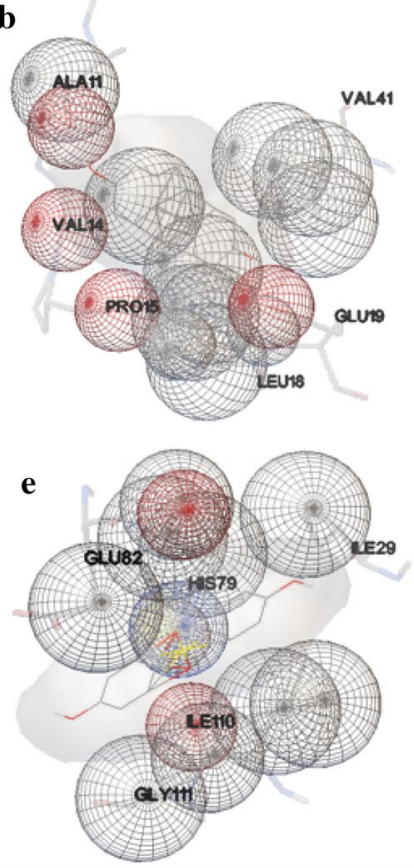
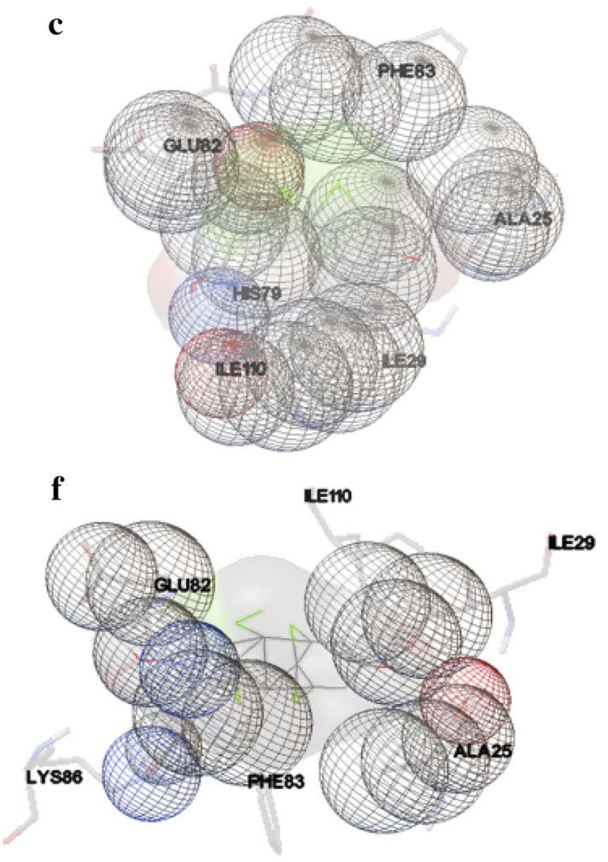

Fig. 4 Binding interactions of the protein TRP 2 with ligands: a BPA, b BPB, $\mathbf{c}$ BPC, $\mathbf{d}$ BPE, e BPS, $\mathbf{f}$ BPAF

Table 2 Binding energy, inhibitory constant and the interacting amino acids of TRP 1 with BPA, BPB, BPC, BPE, BPS and BPAF

\begin{tabular}{|c|c|c|c|}
\hline Ligand & $\begin{array}{l}\text { Binding energy } \\
(\mathrm{kcal} / \mathrm{mol})\end{array}$ & $\begin{array}{l}\text { Inhibitory constant } \\
(\mu \mathrm{M})\end{array}$ & Interacting amino acids \\
\hline BPA & -5.38 & $113.29 \mu \mathrm{M}$ & $\begin{array}{l}\text { ALA 24, ALA 25, SER 25, PHE 83, LYS 86, GLU 82, } \\
\text { HIS 79, ILE } 110\end{array}$ \\
\hline ВРB & -5.85 & $51.63 \mu \mathrm{M}$ & ALA 11, VAL 14, PRO 15, LEU 18, VAL 41, GLU 19 \\
\hline $\mathrm{BPC}$ & -7.26 & $4.75 \mu \mathrm{M}$ & GLU 82, HIS 79, ILE 110, ILE 29, ALA 25, PHE 83 \\
\hline $\mathrm{BPE}$ & -6.37 & $21.54 \mu \mathrm{M}$ & HIS 79, ILE 110, ILE 29, SER 28, ALA 25, PHE 83 \\
\hline BPS & -6.21 & $28.09 \mathrm{M}$ & GLU 82, HIS 79, ILE 29, ILE 110, GLY 111 \\
\hline BPAF & -5.56 & $83.38 \mu \mathrm{M}$ & ALA 25, GLU 82, ILE 11O, ILE 29, LYS 86, PHE 83 \\
\hline
\end{tabular}

were formed between Val 89 and Arg 55 residues of TRP 1 and more effectively binding bisphenols such as BPC, BPB and BPS. The amino acid residues of Ile 110, His 79, Ala 25, Phe 83 of TRP 2 protein are found in common that possessed interaction with almost all bisphenol analogues by means of strong hydrogen bonds that contributed to their effective binding energies and stability. The difference in the binding affinity may also occur probably due to the variation in the 
substitution groups of bisphenol and thus the variations in their structure. This may also cause varied inhibitory effects.

\section{Discussion}

Previous study on the pigmentation interference of bisphenol $\mathrm{F}$ and bisphenol A has revealed that BPA, BPF and PHBPA potentially inhibited the zebra fish and in situ modelled human tyrosinase, TRP1 and TRP2 when analysed by in vitro and in silico methods. The study indicated that BPA inhibited TRP1 with a binding energy of $-5.75 \mathrm{kcal} / \mathrm{mol}$, and TRP2 with that of $-5.87 \mathrm{kcal} / \mathrm{mol}$. In accordance with this data, this study also showed the nearly same binding energy of -5.71 and -5.38 when BPA was docked with TRP1 and TRP2, respectively.

In this study, bisphenol analogues other than BPA that currently serve as an alternative for it have been studied. For the protein TRP1, all bisphenols exhibit high binding affinity comparatively to the predominant and regularly studied BPA. It is a matter of concern that BPS which is an immediate alternative to BPA has a high binding affinity greater than BPA. Bisphenol $\mathrm{C}$ has the binding energy of about $-7.32 \mathrm{kcal} / \mathrm{mol}$ but has the lowest inhibitory constant (Ki) of $2.73 \mu \mathrm{M}$. Of all the compared bisphenols, BPA has been found to exhibit high inhibition of about $64.98 \mu \mathrm{M}$. In terms of showing inhibitory effect on the TRP1 protein, the ligands are in the ascending order of $\mathrm{BPC}<\mathrm{BPB}<\mathrm{BPS}<$ $\mathrm{BPE}<\mathrm{BPAF}<\mathrm{BPA}$.

For the protein TRP 2, bisphenol $\mathrm{C}$ has the binding energy of about $-7.26 \mathrm{kcal} / \mathrm{mol}$ but has the lowest inhibitory constant (Ki) of $4.73 \mu \mathrm{M}$. The same scenario was observed in that of binding with TRP 1 . Similarly, to the values observed in TRP 1, BPA has been found to exhibit high inhibition of about $113.29 \mu \mathrm{M}$. In terms of showing inhibitory effect on the TRP2 protein, the ligands are in the ascending order of $\mathrm{BPC}<\mathrm{BPE}<\mathrm{BPS}<\mathrm{BPB}<\mathrm{BPAF}<\mathrm{BPA}$.

From the results, it is evident that all bisphenol derivatives have an inhibitory effect on the TRP 1 and TRP 2 proteins. In both cases, the inhibitory constant is high for BPA even then if it binds with energy less than that of BPC with high binding energy but showing less inhibition comparatively.

MITF is one of the important proteins that has a regulatory roles with the promotor regions of tyrosinase family proteins such as tyrosinase, TRP 1 . Former study had established that hair dyes resorcinol and lawsone downregulated the MITF expression and resulted in reduced melanin production. Bisphenols are also widely found in hair dyes and colouring agents in food products. In this aspect, there arises a presumption that bisphenols may also have effects on MITF proteins that can eventually lead to depigmentation and related disorders.

\section{Materials and methods}

\section{In silico analysis}

An in silico analysis has been done using AutoDock 4.2.5 which provides good quality docking results with advantages of making sides chains flexible in the protein and shows the interaction between the protein and the ligand. Hence, the molecular docking was performed between proteins TRP1 (PDB id 5M8L), TRP2 (PDB id 1DPT) and bisphenol (BPA-6623, BPB-66166, BPC-84677, BPE608116, BPS-6626 and BPAF-73864) ligands using AutoDock 4.2.5.

\section{Protein preparation}

The 3D structures of all the three protein molecules used for the docking have been downloaded from PDB (https:// www.rcsb.org/) in its default pdb format. The three different proteins which play crucial role in the melanin synthesis have been analysed in this study. The downloaded protein was screened for its active site using Discovery studio visualizer. (https://www.3ds.com/products-services/ biovia/products/molecular-modeling-simulation/bioviadiscovery-studio/). Then, the protein has been prepared for docking which includes, removal of heteroatoms and water molecule. The protein has been added with polar hydrogens and Kollman charges and written in the pdb format.

\section{Ligand preparation}

Six different derivatives of bisphenols, namely BPA, BPB, BPC, BPE, BPS and BPAF, have been docked with the above-mentioned proteins whose 2D structures were downloaded from PubChem (https://pubchem.ncbi.nlm.nih.gov/) in their sdf file formats. The ligands have been converted from sdf file format to pdb file format using Open Babel 2.4.0 for them to be used in AutoDock. The ligand has been prepared for docking by giving them different torsion parameters and required number of torsions has been set and outputs were then saved into pdbqt format.

\section{Grid generation and molecular docking}

From the grid option, the protein molecule in pdb format has been open and then saved in pdbqt format. The pdbqt format ligand has also been opened. Grid box was generated around the active site of the protein for the ligand to dock. The XYZ values of the grid have been assigned based on the values obtained from Discovery studio, and the dimensions were set. The output file was saved in the gpf format. 
After the preparation of the grid file, the protein and the ligand in the pdbqt format have been selected from the docking option. Genetic algorithm was used as the search parameter, and the number of GA runs was set at its default and the maximum value of 10 . Docking parameters were set, and the output file was saved in the dpf format with Lamarckian algorithm. The prepared grid and the dock file were run for analysis.

\section{Conclusion}

From the above study, we can conclude that most of the products we use in our daily life have a high risk of causing various adverse health hazards by getting into us without our consciousness. The predominantly used bisphenol derivative BPA can easily bind to the enzymes with high stability and has the high ability to inhibit the melanogenic enzymes which are very essential in the pigment-producing process. Also, it should be noted that the ligand BPC has a high binding energy and low inhibitory constant. This means that even with small amount of exposure to BPC, the enzymes are prone to inhibition. Their interactions with the TRP1 and TRP2 proteins have been displayed along with their analogues such as BPB, BPS, BPC, BPE and BPAF. The interactions are quantified based on the binding energy and inhibitory constants. There may be difficulties in the synthesis of melanin if the pathway has been affected. And it may also lead to UV damage-based mutations in melanocyte cells, which may affect their ability to serve as protective barrier of DNA and hence may cause tumours such as melanoma. Hence, there is a need for an alternative to bisphenols and its derivatives which should be eco-friendly and safe to use.

Acknowledgements Authors are thankful to B.S. Abdur Rahman Institute of Science \& Technology, Chennai, for providing research facilities in school of life sciences. The authors also gratefully acknowledge the Ministry of Science and Technology, Department of Science and Technology (KIRAN Division) (GoI), New Delhi. (Ref No. DST/WOSB/2018/1583-HFN (G)), DST/SATYAM/COVID-19/2020/213 (G). and ASEAN University network (AUN)/Southeast Asia Engineering Education Development Network (SEED)/Japan International Cooperation Agency (JICA) SPRAC (SN042/MI.KU/2020)

\section{Declarations}

Conflict of interest V Harinishree, Ranjani S, Hemalatha S declare no conflicts of interest.

Ethical approval This article does not contain any studies with human participants or animals performed by any of the authors.

\section{References}

1. Taylor SR (2001) for Corrosion Protection: Organic in Encyclopedia of Materials:Science and Technology $2^{\text {nd }}$ Edn. In: Buscho KHJ, Cahn R, Flemings M, Ilschner B, Kramer E, Mahajan S, Veyssiere P (eds) University of Virginia, Charlottesville, Virginia, USA, pp 1274-1279

2. Konieczna A, Rutkowska A, Rachoń D et al (2015) Health risk of exposure to Bisphenol A (BPA). Rocz Panstw Zakl Hig 66(1):5-11

3. Hélène S, Beausoleil C, Habert R et al (2019) Evidence for bisphenol B endocrine properties. Sci Regul Perspect Environ Health Perspect 127(10):106

4. Liu X, Suyama K, Nose T et al (2021) Bisphenol-C is the strongest bifunctional ER $\alpha$-agonist and ER $\beta$-antagonist due to magnified halogen bonding. PLoS ONE 16(2):e0246583

5. Pozdnyakov P et al (2011) Aqueous Photochemistry of Bisphenol E in the Presence of $\beta$ Cyclodextrin1. High Energy Chem 45(3):214-221

6. Thoene M, Dzika E, Gonkowski S et al (2020) Bisphenol S in food causes hormonal and obesogenic effects comparable to or worse than bisphenol A: a literature review. Nutrients 12(2):532

7. Maćczak A, Cyrkler M, Bukowska B et al (2017) Bisphenol A, bisphenol S, bisphenol $\mathrm{F}$ and bisphenol AF induce different oxidative stress and damage in human red blood cells ( in vitro study). Toxicol In Vitro 41:143-149

8. Bermudez DS, Gray LE Jr, Wilson VS et al (2010) Modeling the interaction of binary and ternary mixtures of estradiol with bisphenol $\mathrm{A}$ and bisphenol AF in an in vitro estrogen-mediated transcriptional activation assay (T47D-KBluc). Toxicol Sci 116(2):477-487

9. Thomas AJ, Erickson CA (2008) The making of a melanocyte: the specification of melanoblasts from the neural crest. Pigment Cell Melanoma Res 21(6):598-610

10. Hirobe T, Shinpo T, Higuchi K, Sano T et al (2010) Life cycle of human melanocytes is regulated by endothelin- 1 and stem cell factor in synergy with cyclic AMP and basic fibroblast growth factor. J Dermatol Sci 57(2):123-131

11. Ando H, Niki Y, Ito M et al (2012) Melanosomes are transferred from melanocytes to keratinocytes through the processes of packaging, release, uptake, and dispersion. J Invest Dermatol 132(4):1222-1229

12. Rzepka Z, Buszman E, Beberok A et al (2016) From tyrosine to melanin: Signaling pathways and factors regulating melanogenesis. Postepy Hig Med Dosw 70:695-708

13. D'Alba L, Shawkey MD (2019) Melanosomes: biogenesis, properties, and evolution of an ancient organelle. Physiol Rev 99(1):1-9

14. Solano F (2014) Melanins: skin pigments and much more-types, structural models, biological functions, and formation routes. New J Sci

15. Mu X, Liu J, Yuan L et al (2020) The pigmentation interference of bisphenol F and bisphenol A. Environ Pollut 266(1):115139 